\title{
Article \\ Induction of Chromosomal Aneuploids from Brewery Shochu Yeast with Novel Brewery Characteristics
}

\author{
Yuki Kusaba ${ }^{1,+}{ }^{,}$Akira Otsuka ${ }^{1,+}$, Huanghuang Dai ${ }^{2}$, Shigeki Inaba ${ }^{3}$ and Hiroshi Kitagaki ${ }^{2,3, * \mathbb{C}}$ \\ 1 Graduate School of Advanced Health Sciences, Saga University, 1 Honjo-cho, Saga 840-8502, Japan; \\ k.msupa.yuki@gmail.com (Y.K.); 21626006@edu.cc.saga-u.ac.jp (A.O.) \\ 2 United Graduate School of Agricultural Science, Kagoshima University, 1-21-24 Korimoto, \\ Kagoshima 890-0065, Japan; 21975002@edu.cc.saga-u.ac.jp \\ 3 Faculty of Agriculture, Saga University, 1 Honjo-cho, Saga 840-8502, Japan; inabas@cc.saga-u.ac.jp \\ * Correspondence: ktgkhrs@cc.saga-u.ac.jp \\ + These authors contributed equally to this work.
}

Citation: Kusaba, Y.; Otsuka, A.; Dai, H.; Inaba, S.; Kitagaki, H. Induction of Chromosomal Aneuploids from Brewery Shochu Yeast with Novel Brewery Characteristics. Fermentation 2022, 8, 62. https://doi.org/10.3390/ fermentation 8020062

Academic Editors: Ogueri Nwaiwu and Mohamed Gedi

Received: 19 December 2021

Accepted: 27 January 2022

Published: 30 January 2022

Publisher's Note: MDPI stays neutral with regard to jurisdictional claims in published maps and institutional affiliations.

Copyright: (C) 2022 by the authors. Licensee MDPI, Basel, Switzerland. This article is an open access article distributed under the terms and conditions of the Creative Commons Attribution (CC BY) license (https:// creativecommons.org/licenses/by/ $4.0 /)$.

\begin{abstract}
The first development method of brewery shochu yeast focusing on chromosomal aneuploidy is reported in this study. Euploidy diploid shochu yeast S-3 was treated with a microtubule inhibitor, nocodazole, for the purpose of inducing aneuploidy. Next, 2,3,5-triphenyl tetrazolium chloride (TTC) staining and the growth rate were investigated to select aneuploids. Aneuploids were selected at a frequency of $8.2 \times 10^{-4}$, which was significantly higher than that of the control group, mainly at chromosomes I, II, III, IX, XII, XIII, and XVI. The acquired aneuploids were evaluated for their metabolic and brewing characteristics. A hierarchical cluster analysis based on endogenous metabolite data discriminated euploid S-3 and aneuploids. In addition, principal-component analysis of the constituents of the broth brewed with the strains discriminated between euploid S-3 and aneuploids. Sensory evaluation of the broth brewed with euploid S-3 and aneuploids showed that it tended to differ in aroma and taste. Specific ethanol production rates of the aneuploids were not deteriorated. The method of this selection made it possible to efficiently obtain aneuploids with various brewing characteristics from brewer's yeast, which do not correspond to genetically modified organisms. This novel breeding method focusing on chromosomal aneuploidy will facilitate the development of novel shochu yeast strains.
\end{abstract}

Keywords: brewery shochu yeast; chromosomal aneuploidy; nocodazole; 2,3,5-triphenyl tetrazolium chloride

\section{Introduction}

Shochu is a traditional Japanese distilled liquor, which started as an industry in the 16th century. It is made by distilling mash containing: koji; several kinds of crops such as barley, rice, and potato; and shochu yeast, Saccharomyces cerevisiae, which is used for ethanol fermentation. Since shochu is mainly produced in the southernmost mainland island of Japan, Kyushu, and Kyushu has a warm and moist climate, the industry has suffered from the proliferation of unfavorable microbes in the mash since its beginning in the 16th century. To combat this problem, a citric acid-producing fungus, Aspergillus luchuensis, was introduced in the early 1900s from the Okinawa islands, which made the mash acidic and prevented the proliferation of unfavorable microbes. To cope with this revolution in the shochu industry, brewery yeasts tolerant to citric acid were isolated [1,2] in the same period and have been utilized until now. Two major shochu yeasts, kyokai yeast for shochu No. 2 (S-2) and kyokai yeast for shochu No. 3 (S-3), belonging to Saccharomyces cerevisiae are commercially available from the Brewing Society of Japan. S-2 was isolated from the mash of Kuma shochu in Kumamoto prefecture [3] and has a favorable fermentation ability. S-3 was isolated from barley koji and has heat and citric acid tolerances [4].

Brewery yeast imparts critical flavors to beverages. Generally, yeasts are diploids and have two sets of chromosomes 1-16, which are called euploids. However, some brewery 
yeasts have three chromosomes and are called aneuploids $[5,6]$. Aneuploidy yeast cells have physiologies that are different from euploidy yeast cells $[7,8]$. Recently, we first showed that the chromosomal aneuploidy of brewery yeasts alters brewery profiles [9]. The breeding of brewery yeast has been attempted first by isolation from nature or favorable mashes [10], and later, through protoplast fusion, the selection of tolerant or sensitive strains and mating. Numerous novel breeding strategies have been developed in sake yeasts [11], but breeding technologies for shochu yeasts have also been developed. These include protoplast fusion and isolating mutants tolerant to heat or $\mathrm{NaCl}$ to produce high-glycerol yeast $[12,13]$. These innovative breeding technologies have led to many industrial revolutions in the sake and shochu industries. However, the changes in the phenotypes were attributed to single nucleotide polymorphism (SNP) [14] or nonreciprocal recombination [15] but not chromosomal aneuploidy.

To develop a method to screen and obtain aneuploids from shochu yeasts, we focused on previous studies on nocodazole. Nocodazole is a synthetic benzimidazole derivative, which interferes with microtubules and was found to inhibit the mitosis of malignant mammalian cells by inhibiting chromosomal segregation [16]. Additionally, it was reported that aneuploidy can be induced in laboratory yeast Saccharomyces cerevisiae [17]. However, this technique has not been applied to brewery yeasts to date.

In order to screen candidate aneuploids, we invented a 2,3,5-triphenyl tetrazolium chloride (TTC) plate technique. TTC was synthesized as a dye for vital tissues [18] and was later utilized to discriminate between brewery yeasts and wild yeasts. TTC itself is white, but when it enters yeast cells, it is reduced by intracellular NADH [19], converted to 1,3,5-Triphenyl formazan (TPF), and exhibits a red color. Therefore, strains that have abundant intracellular NADH, namely strong glycolysis flow, such as sake and shochu yeasts, can use NADH to reduce TTC and will exhibit a red color [19]. On the contrary, strains that have less intracellular NADH, namely weak glycolysis flow, cannot reduce TTC to TPF and will exhibit a white color. Since aneuploids have physiologies different from euploids [20], we hypothesized that white colonies are candidates for the strains, which have weak glycolysis flow and are aneuploids. Even if the developed strains have low ethanol productivity, novel brewery characteristics might compensate for the disadvantage of low ethanol productivity.

In this study, to pioneer the possibility of developing a breeding method focused onto chromosomal aneuploidy in shochu yeast, we investigated the effect of nocodazole on the aneuploidy of shochu yeast and the brewery characteristics of the obtained aneuploids. As a result, we first succeeded in developing a method to induce chromosomal aneuploidy in shochu yeast and to alter its brewery characteristics.

\section{Materials and Methods}

Strains: S-2, S-3, and laboratory yeast BY4743 were used for the study. S-2 and S-3 were obtained from the Brewing Society of Japan, and BY4743 was obtained from EUROSCARF (Scientific Research and Development GmbH, Oberursel, Germany).

Karyotyping: The extraction of genomic DNA and karyotyping was performed according to the previous study $[8,9]$. DNA was extracted using the alkali extraction method. $\mathrm{OD}_{260}$ of extracted DNA was measured using spectrophotometer (Shimadzu, Kyoto, Japan) and adjusted to $0.5 \mathrm{ng} / \mu \mathrm{L}$. Each gene on chromosomes 1 to 16 was quantitated using qRT-PCR with the CFX Connect real-time PCR detection system (Bio-Rad Laboratories, Hercules, CA, USA) using primers described in Supplementary Table S1. Reactions were performed by mixing $5 \mu \mathrm{L}$ KOD SYBR qPCR mix (Toyobo, Osaka, Japan), $0.4 \mu \mathrm{L}$ primer (final $0.4 \mu \mathrm{M}$ ), $2.0 \mu \mathrm{L} 0.5 \mathrm{ng} / \mu \mathrm{L}$ genomic DNA, and $2.2 \mu \mathrm{L}$ Milli-Q (total $10 \mu \mathrm{L}$ ), and by reacting at $95^{\circ} \mathrm{C}$ for $2 \mathrm{~min},\left(95^{\circ} \mathrm{C} 10 \mathrm{~s}, 58^{\circ} \mathrm{C} 30 \mathrm{~s}, 68^{\circ} \mathrm{C} 30 \mathrm{~s}\right) \times 40$ cycles.

Nocodazole treatment: Yeast cells were incubated in YPD medium at $30^{\circ} \mathrm{C}$ overnight. Yeast cells were adjusted to $\mathrm{OD}_{600}=0.1$, dissolved in YPD medium containing $1 \mathrm{ppm}$ nocodazole (FUJIFILM Wako Pure Chemical Co., Osaka, Japan), and incubated at $30^{\circ} \mathrm{C}$ for $4 \mathrm{~h}$. The same procedure was performed without nocodazole and used as a control. 
TTC staining: Yeast cells (1000 cells) were spread onto the TTC lower layer plate (2\% glucose, $0.1 \%$ yeast extract, $0.2 \%$ hipolypeptone, $0.1 \%$ potassium dihydrogen phosphate, $0.04 \%$, magnesium sulfate, and $2.5 \%$ agar) and incubated at $30{ }^{\circ} \mathrm{C}$ for $24 \mathrm{~h}$. Formed white colonies were isolated.

Growth analysis: Isolated colonies were inoculated with YPD medium and incubated at $30{ }^{\circ} \mathrm{C}$ for $24 \mathrm{~h}(n=3)$. Yeast cells were adjusted to $\mathrm{OD}_{600}=0.1$ and incubated at $30{ }^{\circ} \mathrm{C}$ for $6 \mathrm{~h}$. Discriminant analysis of the growth measured by $\mathrm{OD}_{600}$ was performed using the TREND function of Excel. S-3 was used as the euploid, and S-2 was used as the aneuploid.

Extraction of internal metabolites: Yeast cells were incubated in YPD at $30{ }^{\circ} \mathrm{C}$ overnight. Yeast cells corresponding to $4.0 \times 10^{7}$ cells were collected, soaked in liquid nitrogen for $3.5 \mathrm{~min}$, and freeze-dried using a freeze dryer (FDU-1200; EYELA, Tokyo, Japan). Alternatively, $20 \mu \mathrm{L}$ of brewed broth was added to $60 \mu \mathrm{L}$ ribitol (FUJIFILM Wako Pure Chemical Co., Osaka, Japan, $0.2 \mathrm{mg} / \mathrm{mL}$ ) and freeze-dried.

Derivatization: Metabolome analysis using GC-FID was performed as described previously [21]. Methoxyamine solution $(80 \mu \mathrm{L}, 20 \mathrm{mg} / \mathrm{mL}$ dissolved in pyridine) was added to the dried sample and incubated at $30{ }^{\circ} \mathrm{C}$ for $90 \mathrm{~min}$. A $40 \mu \mathrm{L}$ quantity of $n$-methylN-trimethylsilyltrifluoroacetamide (MSTFA; GL Sciences, Tokyo, Japan) was added to the solution, incubated at $37^{\circ} \mathrm{C}$ for $30 \mathrm{~min}$, and applied to GC-FID.

GC-FID analysis: GC-FID analysis was performed using GC-2014 (Shimadzu Co. Ltd., Kyoto, Japan), and the condition is described in Supplementary Table S2. Peaks were identified using standard substances, as described previously [9,22]. Retention times of the metabolites are described in Supplementary Table S3.

Statistical analysis of the metabolites: The peaks of the metabolites detected by GCFID were quantitated using LabSolutions (Shimadzu Co. Ltd., Kyoto, Japan). The area of each peak was normalized according to the total areas of all peaks. Hierarchical clustering was performed using MetaboAnalyst 5.0. Principal component analysis was performed using SIMCAP + v13.0 (Umetrics, Umeå, Sweden) as previously described [9,17].

Small-scale brewing: Small-scale brewing was performed using the candidate yeast strains. In the primary mash, $37.5 \mathrm{~g}$ of shochu koji (Tokushima Seikiku Co., Osaka, Japan), $50 \mathrm{~mL}$ of distilled water, and yeast cells grown in YPD at $30^{\circ} \mathrm{C}$ for $24 \mathrm{~h}$ were mixed and incubated statically at $30^{\circ} \mathrm{C}$ for 5 days. In the secondary mash, $150 \mathrm{~g}$ of steamed barley and $150 \mathrm{~mL}$ of distilled water were added and incubated statically at $30^{\circ} \mathrm{C}$ for 9 days.

Flavor analysis of the brewed broth: Flavor analysis of the brewed broth was performed by transferring $2 \mathrm{~mL}$ of the brewed broth to a $10 \mathrm{~mL}$ vial, and $20 \mu \mathrm{L}$ of acetone was added as an internal standard. The samples were heated using a hot plate stirrer (PCD-610D; TAITEC Co., Saitama, Japan) at $100{ }^{\circ} \mathrm{C}$ for $15 \mathrm{~min}$. SPME fiber (100 $\mu \mathrm{m}$ PDMS; Sigma-Aldrich, St. Louis, MO, USA) was inserted into the sample, held for $10 \mathrm{~min}$, and inserted into the GC system.

Sensory evaluation of broth brewed with the obtained aneuploids: Sample evaluation was performed using quantitative descriptive analysis (QDA) as previously described. Since distillation manipulations of the broth generate experimental deviations, which hamper statistical calculations, sensory evaluation was performed using the broth itself without distillation. The broth was centrifuged and the supernatant was collected. The supernatant was subjected to sensory analysis by trained panels. Two samples were displayed to 9 panels, and descriptions were raised. Through discussion, characteristics that were common to panelists were adopted [16]. Each sample was provided to the panelists, and each characteristic was evaluated with 5 scales (0: weak, 4: strong). Statistical analysis (Dunnett's test) was performed using SPSS $12.0 \mathrm{~J}$ for Windows. $p$-values smaller than 0.2 were described as indicating the existence of a tendency.

\section{Results}

\subsection{Selection of Candidate Aneuploids from Shochu Yeast Using Nocodazole}

In order to screen candidate aneuploids, euploid S-3 was treated with or without nocodazole, and white colonies, which were formed on TTC plates, were picked up. Next, 
strains that showed growth rates at $6 \mathrm{~h}$ incubation in YPD medium lower than the parent strain S-3 were selected. Aneuploidy chromosomes were investigated using qRT-PCR. It turned out that as relative to euploidy S-3 (Figure 1A), chromosome II (anp2, Figure 1B), chromosome IX (anp5, Figure 1C), chromosome III (anp10, Figure 1D), chromosomes IX and XII (anp13, Figure 1E), chromosome XII (anp24, Figure 1F), chromosome IX (anp27, Figure 1G), chromosome XII (anp29, Figure 1H), chromosome XIII (anp30, Figure 1I), chromosome I (anp32, Figure 1J), chromosome XII (anp46, Figure 1K), chromosome XVI (anp59, Figure 1L), and chromosomes IV, V, XI, XIII, and XV (anp101, Figure 1M) were aneuploidy.

qRT-PCR of genomes extracted from each strain was performed, and relative copy numbers of the chromosomes were quantitated using the $\Delta \Delta \mathrm{Ct}$ method and laboratory strain BY4743 as a control. The results are mean values \pm standard deviations of two independent experiments. (A) S-3, (B) anp2, (C) anp5, (D) anp10, (E) anp13, (F) anp24, (G) anp27, (H) anp29, (I) anp30, (J) anp32, (K) np46, (L) anp59, and (M) anp101 were derived from S-3.

The frequency of acquisition of candidate aneuploids was $6.7 \times 10^{-5}\left( \pm 1.6 \times 10^{-5}\right)$ without nocodazole and $8.2 \times 10^{-4}\left( \pm 7.0 \times 10^{-5}\right)$ with nocodazole (Table 1$)$, indicating that the frequency of aneuploidy acquisition tended to be higher when nocodazole is used $(p<0.069)$. Hartwell et al. reported that the frequency of aneuploid acquisition was $2.0 \times 10^{-5}$ [23], indicating that this method using nocodazole is an efficient method to acquire aneuploids. This result indicates that it is possible to acquire chromosome aneuploids using this method.

(A)

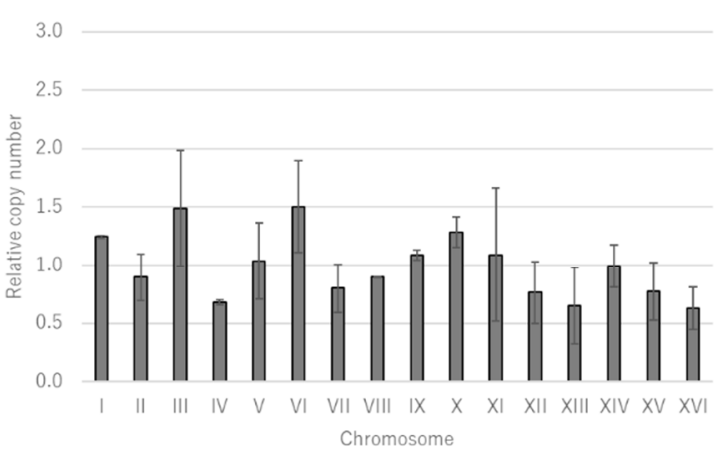

(C)

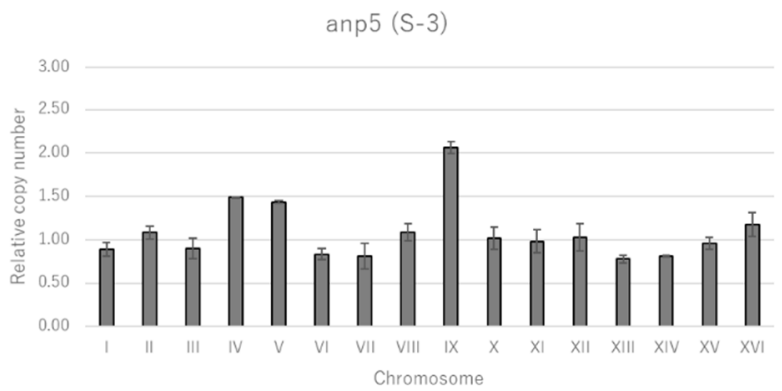

Figure 1. Cont.
(B)

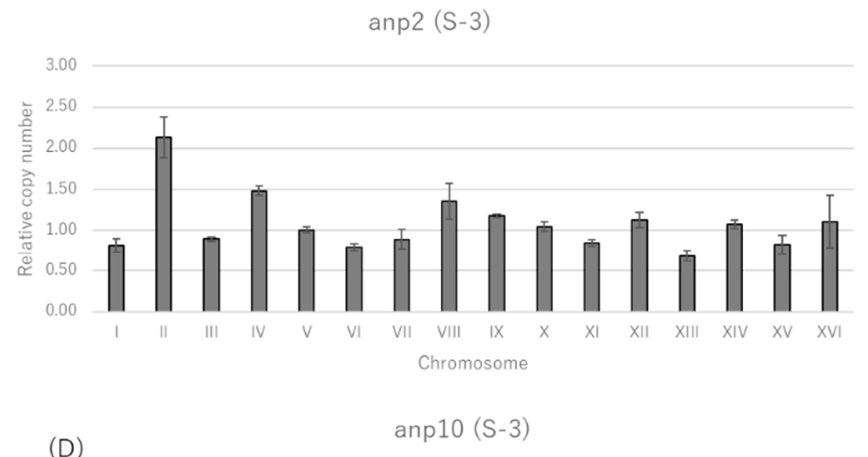

(D)

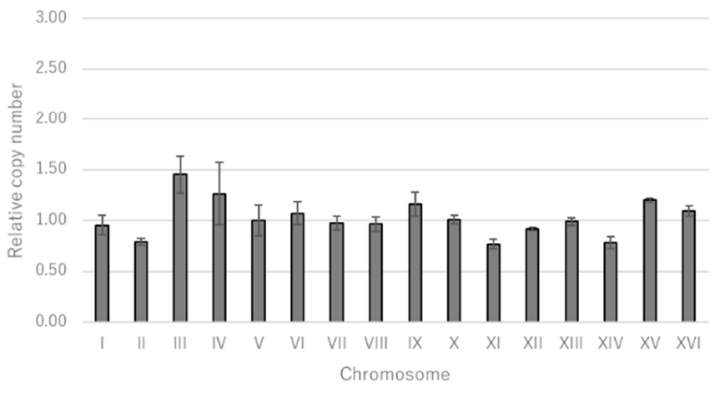


(E)

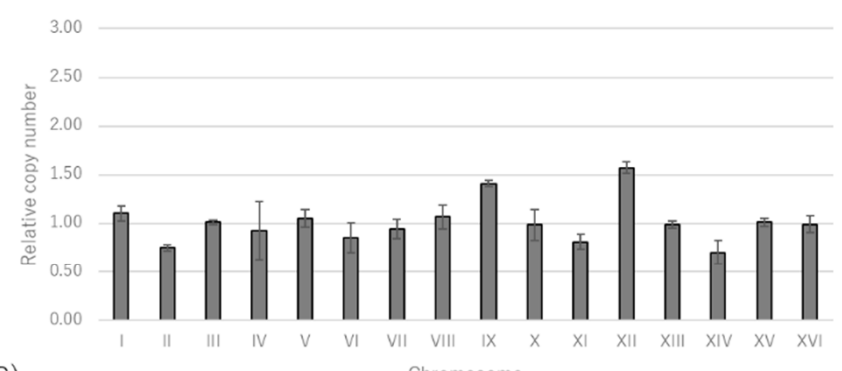

(G)

anp27 (S-3)

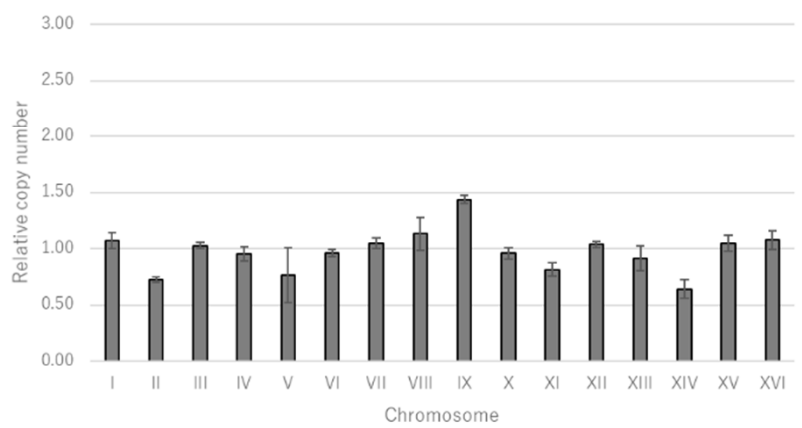

(I)

anp30 (S-3)

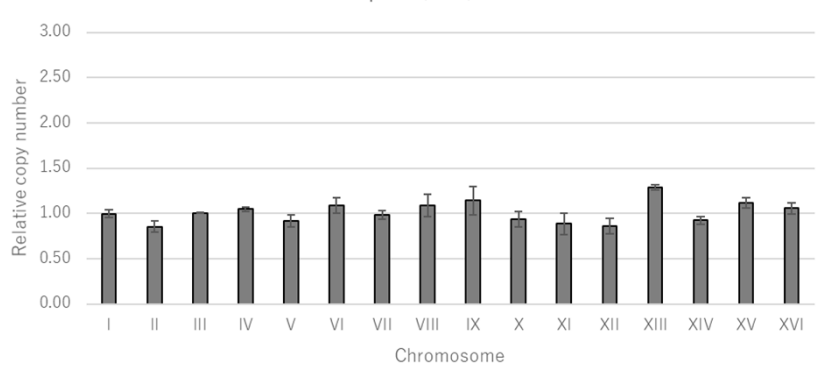

(K)

anp46 (S-3)

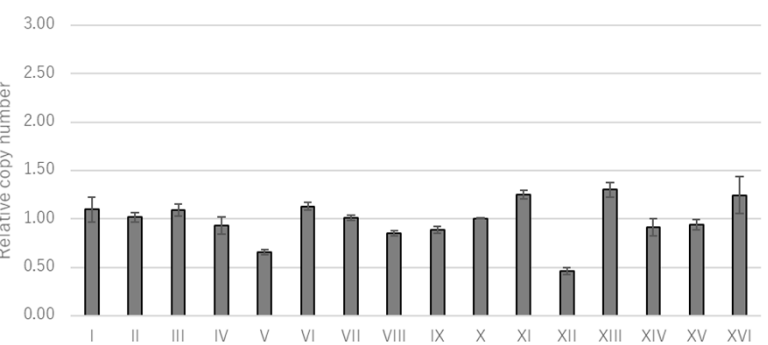

Chromosome
(F)

$\operatorname{anp} 24(\mathrm{~S}-3)$

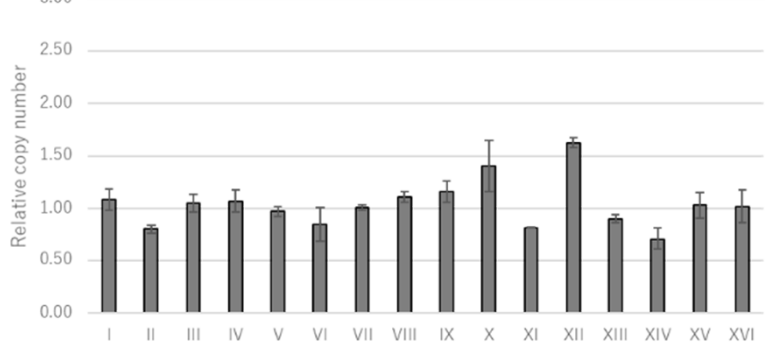

(H)

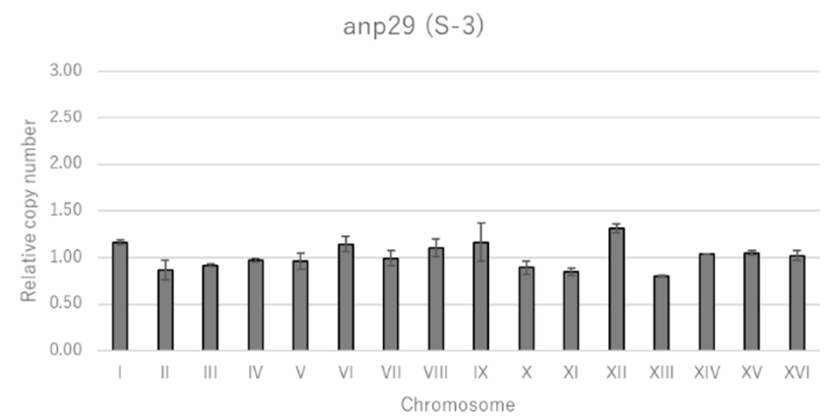

(J)

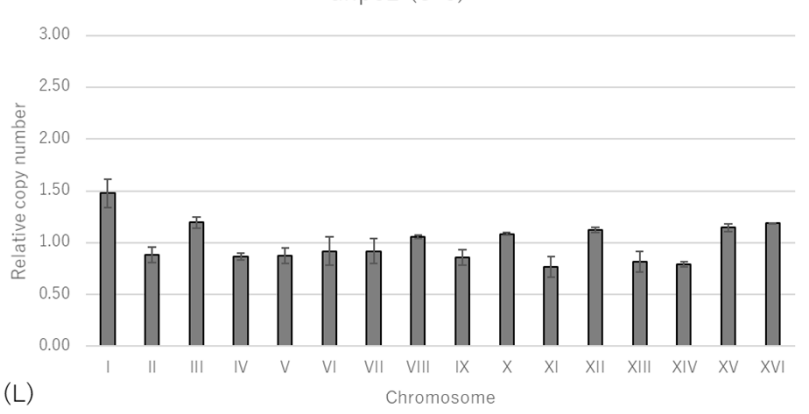

anp59 (S-3)

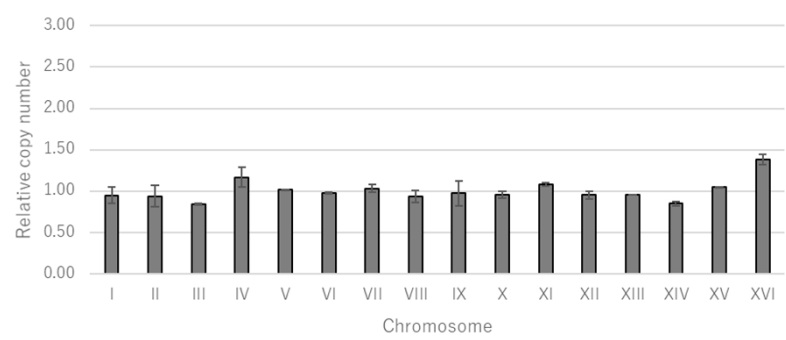

Figure 1. Cont. 
(M)

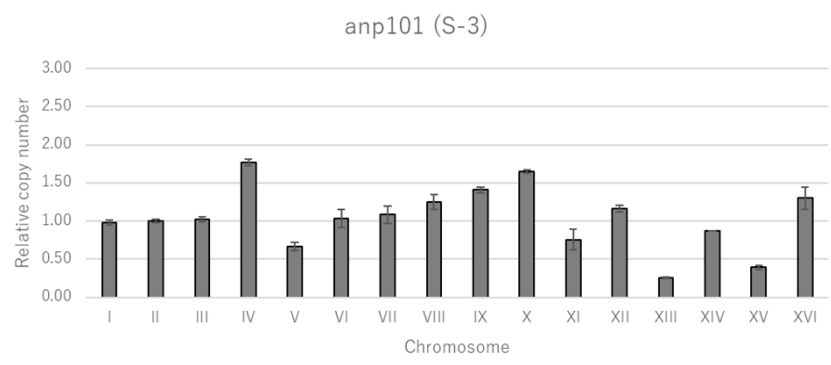

Figure 1. Karyotyping of euploid S-3 and candidate aneuploids derived from S-3. Genomic DNA was extracted, and respective chromosomes were quantitated using qRT-PCR as described in Materials and methods. (A) S-3, (B) anp2 (S-3), (C) anp5 (S-3), (D) anp10 (S-3), (E) anp13 (S-3), (F) anp24 (S-3), (G) anp27 (S-3), (H) anp29 (S-3), (I) anp30 (S-3), (J) anp32 (S-3), (K) anp46 (S-3), (L) anp59 (S-3) and (M) $\operatorname{anp101}(\mathrm{S}-3)$.

Table 1. Frequency of acquisition of aneuploids.

\begin{tabular}{ccccccc}
\hline & \multicolumn{7}{c}{ Nocodazole } \\
\cline { 2 - 7 } & \multicolumn{7}{c}{ Untreated (Control) } & \multicolumn{3}{c}{ Treated } \\
\hline & $\mathbf{1 s t}$ & 2nd & 3rd & 1st & 2nd & 3rd \\
\hline Colony-forming unit & 5000 & 5000 & 5000 & 3000 & 5000 & 5000 \\
TTC straining & 12 & 2 & 6 & 8 & 36 & 30 \\
Growth rate survey & 1 & 2 & 5 & 4 & 12 & 21 \\
Karyotype analysis & 1 & 0 & 0 & 2 & 7 & 2 \\
Appearance frequency & $2.0 \times 10^{-4}$ & 0 & 0 & $6.7 \times 10^{-4}$ & $1.4 \times 10^{-3}$ & $4.0 \times 10^{-4}$ \\
of aneuploids & & & & & & \\
\hline Mean $( \pm$ SE $)$ & $6.7 \times 10^{-5}\left( \pm 1.6 \times 10^{-5}\right)$ & $8.2 \times 10^{-4}\left( \pm 7.0 \times 10^{-5}\right) *$ \\
\hline
\end{tabular}

Appearance frequency of aneuploids was calculated by dividing the number of aneuploids obtained by karyotype analysis by the value of colony-forming unit. Statistical differences were judged by an unpaired two-sided Student's $t$-test. ${ }^{*} p<0.1$.

\subsection{Metabolome Analysis of the Broth Brewed with the Obtained Aneuploids}

In order to investigate if the intracellular metabolites of the aneuploids are different from those of euploids, metabolome analyses of the intracellular metabolites of the nine acquired aneuploids were performed. Shochu yeast S-2 was used for subsequent analyses as a reference. Hierarchical clustering analysis (HCA) indicated that the aneuploids were clustered distantly from their parent strains (Figure 2A), indicating that the aneuploid has metabolites different from those of the parent strain.

The principal component analysis (PCA) of the metabolites indicated that the contributions of PC1 and PC2 are $23.4 \%$ and $22.3 \%$, respectively. In the score plot, euploid S-3 was located on the plus side, and aneuploid strains were located on the minus side of PC1 (Figure 2B). In the loading plot, glucose, pyruvate, and citrate were located on the plus side of PC1, and glycerol was located on the plus side of PC1 (Figure 2C). 


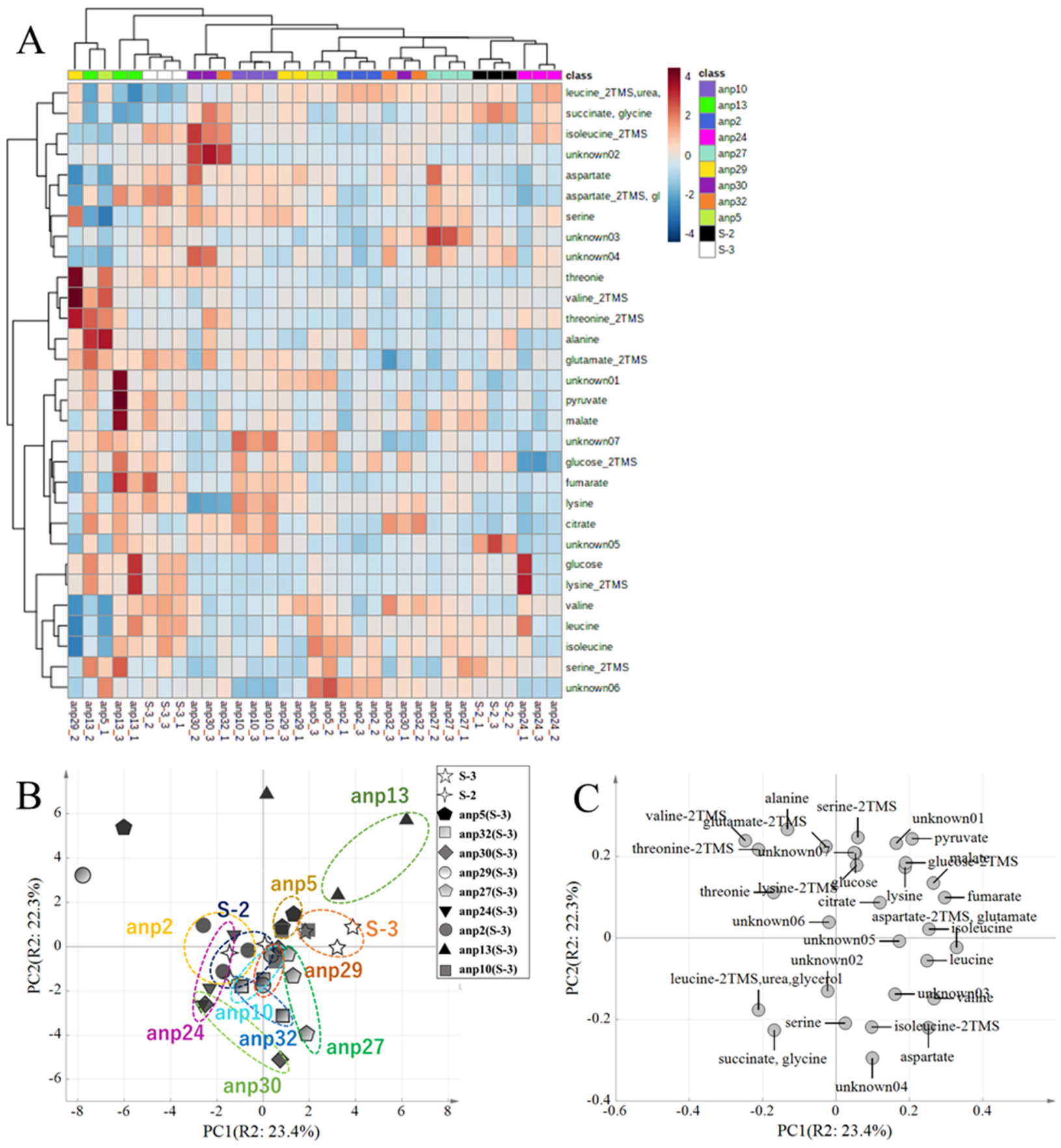

Figure 2. HCA and PCA of endogenous metabolite profiles of shochu yeasts (euploid S-3, aneuploid S-2, aneuploids derived from euploid S-3). (A) HCA of endogenous metabolite profiles of 11 samples. (B) Score plot of PCA (PC1, R ${ }^{2}(23.4 \%) ;$ PC2, $\mathrm{R}^{2}(22.3 \%)$ ). (C) Loading plot of PCA (30 endogenous metabolites). Data were calculated from independent triplicate experiments.

In the preceding experiments, anp13 (derived from euploid S-3) and anp24 (derived from euploid S-3), which were located far away from other strains, were selected and used for the brewing test. Broths brewed with these mutants were analyzed using GC-FID, and the obtained data were analyzed using HCA and PCA. HCA indicated S3-derived aneuploids were clustered distantly from their parental strain S-3 (Figure 3A). These results indicated that the obtained aneuploids have different brewery characteristics. PCA indicated that the aneuploids were located on the plus side of PC2 $\left(\mathrm{R}^{2}=23.9 \%\right)$ and euploid S-3 was located on the minus side of PC2 (Figure 3B). 

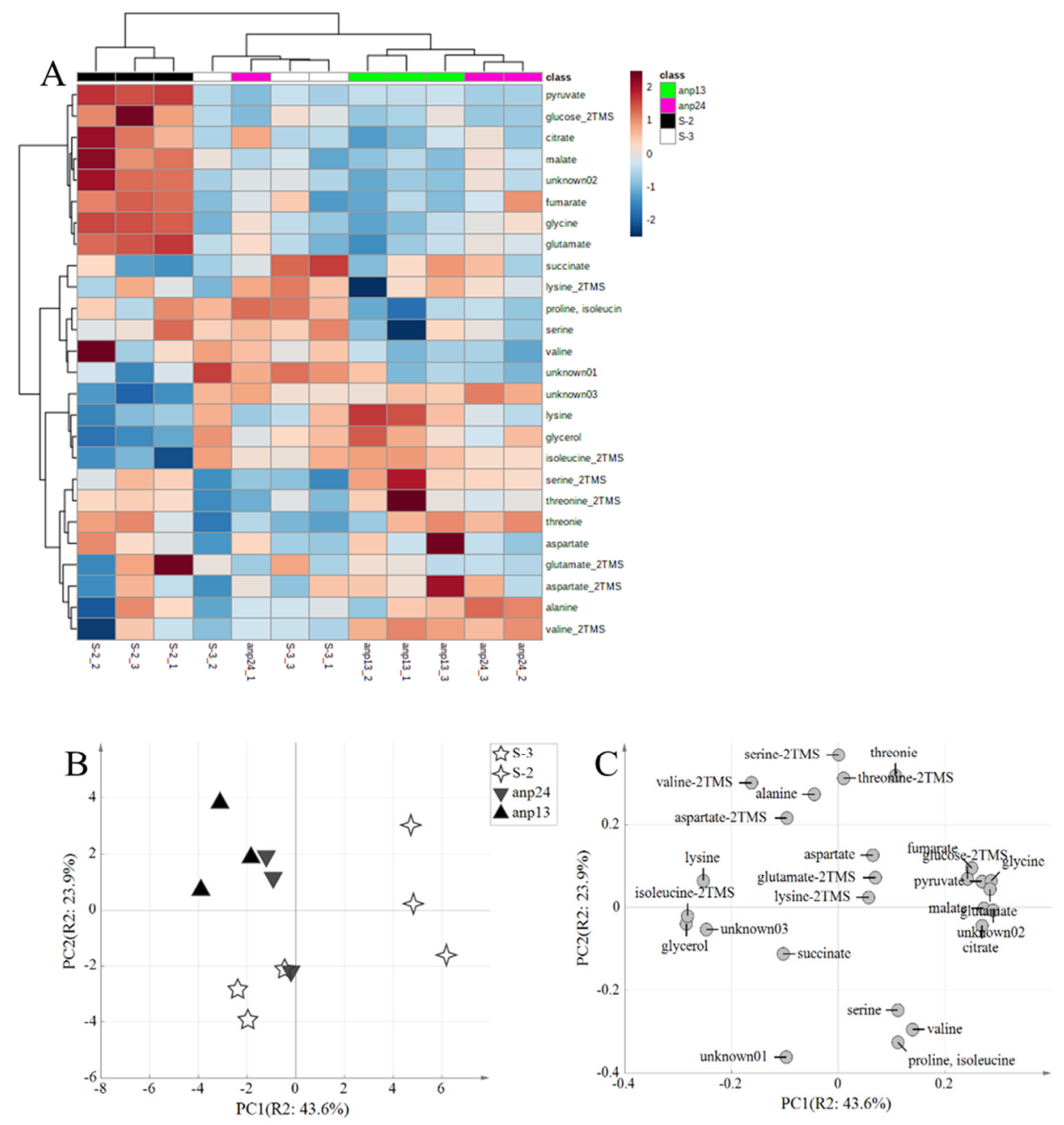

Figure 3. HCA and PCA of compound profiles of the broth brewed with shochu yeasts (euploid S-3, aneuploid S-2, aneuploids 13 and 24, derived from euploid S-3). (A) HCA of hydrophilic metabolite profiles of 4 samples. (B) Score plot of PCA (PC1, R $\mathrm{R}^{2}(37.5 \%)$; PC2, $\mathrm{R}^{2}(24.7 \%)$ ). (C) Loading plot of PCA (25 hydrophilic metabolites). Data were calculated from independent triplicate experiments.

A loading plot showed that aspartic acid, alanine, and threonine are located on the plus side of PC2, and proline was located on the minus side of PC2 (Figure 3C).

\subsection{Flavor Analysis of the Broth Brewed with the Obtained Aneuploids}

In order to evaluate the flavor profiles of the broth brewed with obtained aneuploids, their flavors were analyzed using head space gas chromatography. The HCA of the obtained flavor profiles indicated that aneuploids were clustered distantly from their parent strain, euploid S-3 (Figure 4A). PCA indicated that anp13 (derived from euploid S-3) was scattered on the plus side of PC1 and anp24 (derived from euploid S-3) on the minus side of PC1 in the score plot (Figure 4B). In the loading plot, ethanol was located on the plus side, and 2-metyl1-propanol and 3-methyl-1-butanol were located on the minus side of PC1 (Figure 4C). These results indicated that the aneuploids have different flavor characteristics. 

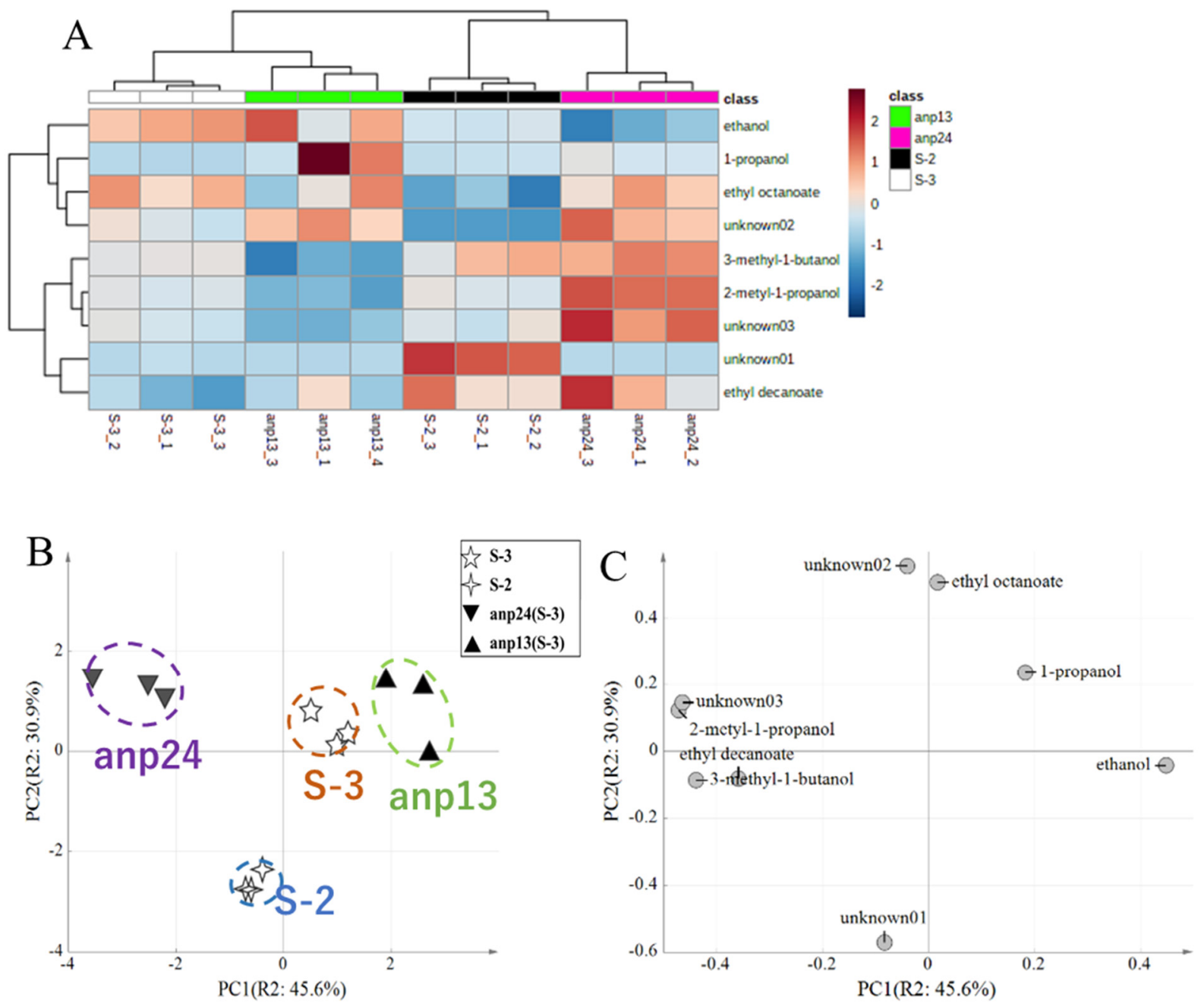

Figure 4. HCA and PCA of volatile profiles of the broth brewed with shochu yeasts (euploid S-3, aneuploid S-2, aneuploids 13 and 24, derived from euploid S-3). (A) HCA of volatiles profiles of 4 samples. (B) Score plot of PCA (PC1, R ${ }^{2}(45.6 \%)$; PC2, $\mathrm{R}^{2}(30.9 \%)$ ). (C) Loading plot of PCA (9 volatile components). Data were calculated from independent triplicate experiments.

\subsection{Sensory Evaluation of Broth Brewed with the Obtained Aneuploids}

To obtain insight into the taste of the broth brewed with the aneuploids, sensory evaluation using the QDA method was performed. Broth brewed with anp13 (derived from euploid S-3) had significantly decreased astringency ( $n=9, p=0.064$, Dunnett's test) and tended to show an increased lemon-like flavor $(p=0.101)$ relative to its parent strain euploid S-3 (Figure 5).

Since ethanol productivity is an important profile of yeast in the distilled liquor industry, ethanol production rates and glucose consumption rates were evaluated (Table 2). It turned out that specific growth rates, specific glucose consumption rates, or the glucose consumption on the yields of biomass of the aneuploids are not altered in relation to its parent strain S-3. The ethanol production on the yields of biomass of anp24 was improved (7.6\% increase in anp24; $p<0.01)$. However, perhaps due to the selection criteria, rates of biomass yields were decreased (11\% decrease in anp13; $p<0.1)$. In total, the obtained aneuploids were considered to be practical in the distilled liquor industry. 


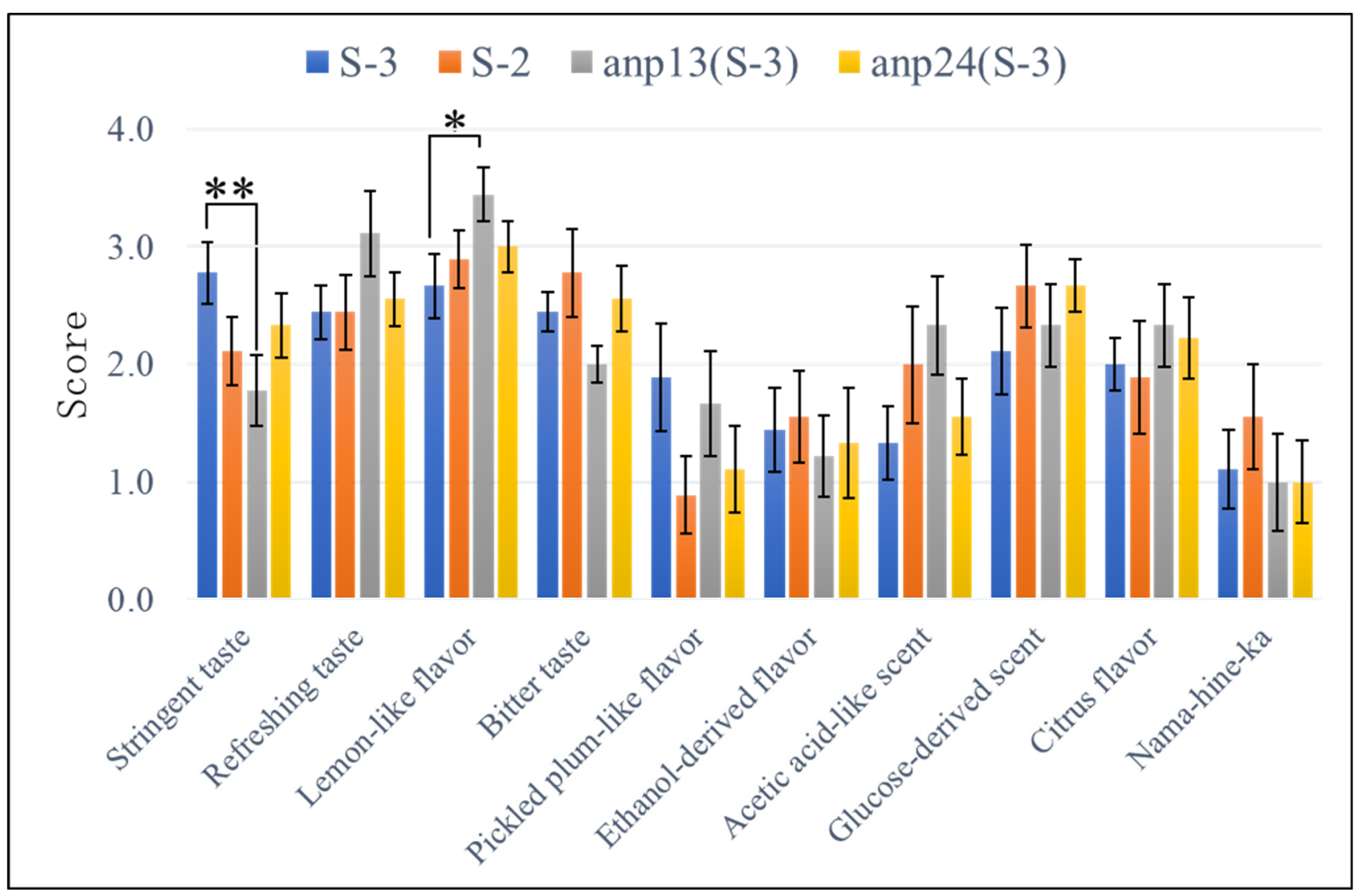

Figure 5. Quantitative descriptive analysis for flavor quality of the broth brewed with shochu yeasts. Statistical differences were judged by Dunnett's test. ${ }^{*} p<0.2,{ }^{* *} p<0.1$. Data were calculated from independent triplicate experiments.

Table 2. Fermentation characteristics of aneuploids cultured in YPD (10\% glucose) medium.

\begin{tabular}{|c|c|c|c|c|c|c|}
\hline & \multicolumn{6}{|c|}{ Mean \pm SEM $^{a}$} \\
\hline & $\mu\left(h^{-1}\right)$ & $\begin{array}{c}\text { Qs } \\
\left(\mathrm{gg}^{-1} h^{-1}\right)\end{array}$ & $\underset{\left(\mathrm{gg}^{-1} h^{-1}\right)}{\text { Qetoh }}$ & Qs/x $\left(\mathrm{gg}^{-1}\right)$ & Qetoh/x $\left(\mathrm{gg}^{-1}\right)$ & CDM $\left(h^{-1}\right)$ \\
\hline S-3 & $0.16 \pm 0.01$ & $1.15 \pm 0.21$ & $1.23 \pm 0.02$ & $55.32 \pm 10.03$ & $58.86 \pm 1.12$ & $0.84 \pm 0.04$ \\
\hline $\mathrm{S}-2$ & $0.13 \pm 0.01$ * & $1.42 \pm 0.09$ & $1.43 \pm 0.03^{* * *}$ & $68.83 \pm 4.42$ & $68.83 \pm 1.24^{* * *}$ & $0.69 \pm 0.02^{* * *}$ \\
\hline anp13 (S-3) & $0.15 \pm 0.00$ & $1.47 \pm 0.10$ & $1.32 \pm 0.02$ & $70.77 \pm 4.69$ & $63.20 \pm 1.15$ & $0.75 \pm 0.01$ * \\
\hline anp24 (S-3) & $0.16 \pm 0.01$ & $1.25 \pm 0.18$ & $1.38 \pm 0.05^{* *}$ & $60.07 \pm 8.76$ & $66.34 \pm 2.61 * *$ & $0.78 \pm 0.02$ \\
\hline
\end{tabular}

The calculation for ethanol production rate and glucose production rate were performed according to the method of Pflügl et al. [24]. Three replicates were used for each strain. Statistical differences were judged by Dunnett's test. ${ }^{*}, p<0.1 ;{ }^{* *}, p<0.05 ;{ }^{* *}, p<0.01$. ${ }^{\text {a }}$ Data are mean values of three independent culture experiments. M $\left(\mathrm{h}^{-1}\right)$, specific growth rates; Qs $\left(\mathrm{gg}^{-1} \mathrm{~h}^{-1}\right)$, specific glucose consumption rates; Qetoh $\left(\mathrm{gg}^{-1} \mathrm{~h}^{-1}\right)$, specific ethanol production rates; Qs $/ x\left(\mathrm{gg}^{-1}\right)$, glucose consumption on the yields of biomass; Qetoh/x ( $\left.\mathrm{gg}^{-1}\right)$, ethanol production on the yields of biomass; CDM $\left(\mathrm{h}^{-1}\right)$, rates of biomass yields.

\section{Discussion}

The breeding of brewery yeasts has been performed through mating, and later, through mutations involving SNPs [14] and nonreciprocal recombination [15]. However, although chromosomal aneuploidy has been observed in many brewery yeasts, the breeding of brewery yeasts involving chromosomal aneuploidy has not been reported. We recently reported that chromosomal aneuploidy alters the brewery characteristics of sake yeast [9]. Therefore, it was considered possible to develop strains with novel characteristics by selecting aneuploids. In this study, by adopting a nocodazole approach, we attempted to obtain aneuploids of shochu yeast with altered brewing characteristics. As a result, aneuploids were efficiently obtained from shochu yeast, and the obtained aneuploids had different brewery characteristics. These results show that screening for aneuploidy strains is a novel and efficient method to obtain novel brewery yeasts. 
Chromosomal aneuploidy is considered to be caused by chromosomal segregation errors during mitosis, such as the problematic attachment of sister chromatids or separase that cleaves the attachment $[25,26]$. Since brewery yeasts have long been maintained in a non-severe condition for generations, it is considered that brewery yeasts may have mutations in some of the genes involved in chromosomal segregation and, as a result, may have generated aneuploids at a high frequency. The mutation responsible for the missegregation of chromosomes to generate aneuploids is a target of future studies.

The fact that chromosomal aneuploidy caused an alteration of the brewery characteristics of shochu yeasts is considered to be caused by the increased expression of specific genes. Indeed, the increased expression of the mitochondrial cytochrome c peroxidase gene, $C C P 1$, contributed to the pyruvate decrease in aneuploid sake yeast [27]. Therefore, the altered brewery characteristics of aneuploids observed in this study is also considered to be the result of the increased expression of specific genes on the increased chromosome, which will be a target of future studies.

The results obtained in this study indicate that chromosomal aneuploidy is an important approach to breeding novel shochu yeast strains. Since checking aneuploidy using qRT-PCR is efficient, checking the copy number of brewery yeasts will be a normal method for the strain selection of brewery yeasts with novel flavors. It should be noted that chromosomal aneuploidy might be unstable. We presently do not have enough data on the stability of the aneuploidy chromosomes (the aneuploidy chromosomes were stable after 100 generations in the culture of YPD, but this might depend on the culture media and subtle mitosis conditions). However, considering that it has been reported that brewery yeasts around the world are chromosomal aneuploids [5,6], it is possible to consider that at least some of the aneuploidy chromosomes can be stably maintained.

Some amino acids are converted to alcohols during brewing [28]. Furthermore, some amino acids are converted to aldehydes through Strecker degradation during distillation [24,29]. Therefore, the alteration of amino acids elucidated in this study might cause changes in the taste of shochu.

In summary, we first developed a screening method for aneuploids in shochu brewery yeast using nocodazole and confirmed that they have novel flavors. This novel method should pioneer a breeding strategy for shochu and related brewery yeasts, which do not correspond to genetically modified organisms.

Supplementary Materials: The following supporting information can be downloaded at: https: / / www.mdpi.com/article/10.3390/fermentation8020062/s1.

Author Contributions: Conceptualization, H.K. and Y.K.; Formal analysis: Y.K., A.O. and H.D. Funding acquisition, H.K.; Investigation, H.K.; Methodology, H.K.; Project administration, H.K. and S.I.; Supervision, H.K. and S.I.; Writing-Original draft, Y.K.; Writing—review and editing, H.K.; All authors have read and agreed to the published version of the manuscript.

Funding: Part of this study was financially supported by JSPS KAKENHI 15K07363 to H.K.

Institutional Review Board Statement: Not applicable.

Informed Consent Statement: Not applicable.

Data Availability Statement: Not applicable.

Acknowledgments: We would like to acknowledge Noriko Ryuda and Seiga Taguchi for their technical assistance and Kazuro Fukuda at Asahi Brewery for his valuable discussion of the breeding methodology.

Conflicts of Interest: The authors declare there are no conflict of interest that could emanate from the publication of this manuscript.

\section{References}

1. Inui, T. Ryukyu awamori hakko kin chosa hokokusho. J. Chem. Soc. Jpn. 1901, 4, 1421-1430. (In Japanese)

2. Usami, K. Awamorishu jozo kenkyu hokoku. Tokyo Univ. Bull. 1901, 3, 15-37. (In Japanese) 
3. Sugama, S. Shochu yeast. J. Brew. Soc. Jpn. 1972, 67, 672-677. (In Japanese)

4. Yoshidome, T.; Maki, N.; Yonetamari, T.; Yoshida, K.; Nakahara, K. Traditional shochu brewing using dry shochu-yeast. J. Brew. Soc. Jpn. 2001, 96, 433-439. (In Japanese) [CrossRef]

5. Gallone, B.; Steensels, J.; Prahl, T.; Soriaga, L.; Saels, V.; Herrera-Malaver, B.; Merlevede, A.; Roncoroni, M.; Voordeckers, K.; Miraglia, L.; et al. Domestication and divergence of Saccharomyces cerevisiae beer yeasts. Cell 2016, 166, 1397-1410.e16. [CrossRef]

6. Peter, J.; Chiara, D.M.; Friedrich, A.; Yue, X.J.; Pflieger, D.; Bergström, A.; Sigwalt, A.; Barre, B.; Freel, K.; Llored, A.; et al. Genome evolution across 1011 Saccharomyces cerevisiae isolates. Nature 2018, 556, 339-344. [CrossRef] [PubMed]

7. Pavelka, N.; Rancati, G.; Zhu, J.; Bradford, W.D.; Saraf, A.; Florens, L.; Sanderson, B.W.; Hattem, G.L.; Li, R. Aneuploidy confers quantitative proteome changes and phenotypic variation in budding yeast. Nature 2010, 468, 321-325. [CrossRef] [PubMed]

8. Fukuda, N.; Honda, S.; Fujiwara, M.; Yoshimura, Y.; Nakamura, T. Polyploid engineering by increasing mutant gene dosage in yeasts. Microb. Biotechnol. 2021, 14, 979-992. [CrossRef] [PubMed]

9. Kadowaki, M.; Fujimaru, Y.; Taguchi, S.; Ferdouse, J.; Sawada, K.; Kimura, Y.; Terasawa, Y.; Agrimi, G.; Anai, T.; Noguchi, H.; et al. Chromosomal aneuploidy improves the brewing characteristics of sake yeast. Appl. Environ. Microbiol. 2017, 83, e01620-17. [CrossRef]

10. Takashita, H.; Fujihara, E.; Furutera, M.; Kajiwara, Y.; Shimoda, M.; Matsuoka, M.; Ogawa, T.; Kawamoto, S.; Ono, K. Competitive advantage and tolerance of selected shochu yeast in barley shochu mash. J. Biosci. Bioeng. 2013, 116, 79-84. [CrossRef]

11. Kitagaki, H.; Kitamoto, K. Breeding research on sake yeasts in Japan: History, recent technological advances, and future perspectives. Annu. Rev. Food Sci. 2013, 4, 215-235. [CrossRef] [PubMed]

12. Omori, T.; Umemoto, Y.; Ogawa, K.; Kajiwara, Y.; Shimoda, M.; Wada, H. A novel method for screening high glycerol- and ester-producing brewing yeasts (Saccharomyces cerevisiae) by heat shock treatment. J. Ferment. Bioeng. 1997, 83, 64-69. [CrossRef]

13. Omori, T.; Ogawa, K.; Shimoda, M. Breeding of high glycerol-producing shochu yeast (Saccharomyces cerevisiae) with acquired salt tolerance. J. Ferment. Bioeng. 1995, 79, 560-565. [CrossRef]

14. Kanai, M.; Kawata, T.; Yoshida, Y.; Kita, Y.; Ogawa, T.; Mizunuma, M.; Watanabe, D.; Shimoi, H.; Mizuno, A.; Yamada, O.; et al Sake yeast YHR032W/ERC1 haplotype contributes to high S-adenosylmethionine accumulation in sake yeast strains. J. Biosci. Bioeng. 2017, 123, 8-14. [CrossRef]

15. Miyashita, K.; Sakamoto, K.; Kitagaki, H.; Iwashita, K.; Ito, K.; Shimoi, H. Cloning and analysis of the AWA1 gene of a nonfoaming mutant of a sake yeast. J. Biosci. Bioeng. 2004, 97, 14-18. [CrossRef]

16. Mimura, N.; Isogai, A.; Iwashita, K.; Bamba, T.; Fukusaki, E. Gas chromatography/mass spectrometry based component profiling and quality prediction for Japanese sake. J. Biosci. Bioeng. 2014, 118, 406-414. [CrossRef]

17. Setoguchi, T.; Kamiwatari, T. General components and general flavor compounds in sweet-potato-shochu and brown-sugarshochu-Analysis of Honkakushochu. J. Brew. Soc. Jpn. 2014, 109, 49-59. (In Japanese) [CrossRef]

18. De Brabander, M.J.; Van de Velre, R.M.L.; Aerts, F.E.M.; Borgers, M.; Janssen, P.A.J. The effects of methyl [5-(2-thienylcarbonyl)1H-benzimidazol-2-yl] carbamate, (R 17934; NSC 238159), a new synthetic antitumoral drug interfering with microtubules, on mammalian cells cultured in vitro. Cancer Res. 1976, 36, 905-916.

19. Zimmermann, F.K.; Mayer, V.W.; Scheel, I. Induction of aneuploidy by oncodazole (nocodazole), an anti-tubulin agent, and acetone. Mutat. Res. Lett. 1984, 141, 15-18. [CrossRef]

20. Mattson, A.M.; Jensen, C.O.; Dutcher, R.A. Triphenyltetrazolium chloride as a dye for vital tissues. Science 1947, 106, 294-295. [CrossRef]

21. Furukawa, T.; Akiyama, H. A microbial control of sake brewing. Part IV. On the TTC agar overlay method for grouping of yeast (1). Nippon Nogeikagaku Kaishi 1963, 37, 398-402. (In Japanese) [CrossRef]

22. Torres, E.M.; Sokolsky, T.; Tucker, C.M.; Chan, L.Y.; Boselli, M.; Dunham, M.J.; Amon, A. Effects of aneuploidy on cellular physiology and cell division in haploid yeast. Science 2007, 317, 916-924. [CrossRef] [PubMed]

23. Hartwell, L.H.; Dutcher, S.K.; Wood, J.S.; Garvik, B. The fidelity of mitotic chromosome reproduction in S. cerevisiae. Rec. Adv. Yeast Mol. BioI. 1982, 1, 28-38.

24. Pflügl, S.; Marx, H.; Mattanovich, D.; Sauer, M. 1,3-Propanediol production from glycerol with Lactobacillus diolivorans. Bioresour. Technol. 2012, 119, 133-140. [CrossRef] [PubMed]

25. Janssen, A.; van der Burg, M.; Szuhai, K.; Kops, G.J.; Medema, R.H. Chromosome segregation errors as a cause of DNA damage and structural chromosome aberrations. Science 2011, 333, 1895-1898. [CrossRef]

26. Makrantoni, V.; Marston, A.L. Cohesin and chromosome segregation. Curr. Biol. 2018, 28, R688-R693. [CrossRef]

27. Fujimaru, Y.; Kusaba, Y.; Zhang, N.; Dai, H.; Yamamoto, Y.; Takasaki, M.; Kakeshita, T.; Kitagaki, H. Extra copy of the mitochondrial cytochrome-c peroxidase gene confers a pyruvate-underproducing characteristic of sake yeast through respiratory metabolism. J. Biosci. Bioeng. 2021, 131, 640-646. [CrossRef]

28. Ough, C.S.; Bell, A.A. Effects of nitrogen fertilization of grapevines on amino acid metabolism and higher-alcohol formation during grape juice fermentation. Am. J. Enol. Vitic. 1980, 31, 122-123.

29. Okumura, J. Maillard reaction and flavor formation. J. Brew. Soc. Jpn. 1993, 88, 178-187. (In Japanese) [CrossRef] 